\title{
Quality Assessment and Usage Behavior of a Mobile Voice-over-IP service.
}

\author{
Toon De Pessemier • Isabelle Stevens • Lieven De Marez • Luc \\ Martens • Wout Joseph
}

Received: date / Accepted: date

\begin{abstract}
Voice-over-IP (VoIP) services offer users a cheap alternative to the traditional mobile operators to make voice calls. Due to the increased capabilities and connectivity of mobile devices, these VoIP services are becoming increasingly popular on the mobile platform. Understanding the user's usage behavior and quality assessment of the VoIP service plays a key role in optimizing the Quality of Experience (QoE) and making the service to succeed or to fail.

By analyzing the usage and quality assessments of a commercial VoIP service, this paper identifies device characteristics, context parameters, and user aspects that influence the usage behavior and experience during VoIP calls. Whereas multimedia services are traditionally evaluated by monitoring usage and quality for a limited number of test subjects and during a limited evaluation period, this study analyzes the service usage and quality assessments of more than thousand users over a period of 120 days. This allows to analyze evolutions in the usage behavior and perceived quality over
\end{abstract}

T. De Pessemier - L. Martens - W. Joseph

iMinds - Ghent University, Dept. of Information Technology, $\mathrm{WiCa}$

G. Crommenlaan 8 box 201, 9050 Ghent, Belgium

Tel.: +32-09-33-14908

Fax: +32-09-33-14899

E-mail: toon.depessemier@ugent.be

E-mail: luc1.martens@ugent.be

E-mail: wout.joseph@ugent.be

I. Stevens - L. De Marez

iMinds - Ghent University, Dept. of Communication Sciences, MICT

Korte Meer 7-9-11, 9000 Ghent, Belgium

Tel.: +32-09-264 9767

E-mail: isabelle.stevens@ugent.be

E-mail: lieven.demarez@ugent.be time, which has not been done up to now for a widelyused, mobile, multimedia service. The results show a significant evolution over time of the number of calls, the call duration, and the quality assessment. The time of the call, the used network, and handovers during the call showed to have a significant influence on the users' quality assessments.

Keywords usage behavior · user experience · voiceover-ip $\cdot$ mobile $\cdot$ quality assessment

\section{Introduction}

Nowadays, a variety of Voice-over-IP (VoIP) services offers users the possibility to make free or cheap voice calls using the Internet. VoIP services such as Skype ${ }^{1}$, $\mathrm{ooVoo}^{2}$, and Google Hangouts ${ }^{3}$ are becoming increasingly popular due to the cost reduction benefit and its flexibility (in contrast to landlines, VoIP is not tied to a specific address) [5]. Most of these services also provide a mobile application, enabling the users to make VoIP calls with their tablet or smartphone. This provides users an alternative for the traditional GSM (Global System for Mobile Communications) standard, i.e., the set of protocols for second generation (2G) digital cellular networks used by mobile phones for telephony. In recent years, cheap data plans of mobile operators and an increased coverage of cellular data networks further stimulated the growing popularity of VoIP applications on mobile devices.

Quality and reliability are two dark spots on the reputation of VoIP multimedia applications. Over the

\footnotetext{
1 http://www.skype.com

2 http://www.oovoo.com

3 http://www.google.com/hangouts
} 
years, there has been much improvement due to better networks and audio codecs. But still, people are very demanding regarding voice quality in VoIP because they are used for years to the impeccable quality of landline phones [1].

As a result, end-to-end Quality of Service (QoS) management is becoming a challenge [24] in order to provide high quality and reliable communication over a best-effort network. Unfortunately, the QoS parameters do not always reflect the perceived quality of a service perfectly, since they only take into account network related aspects and neglect device characteristics, context parameters, and user aspects. In contrast, subjective quality measurements with actual test subjects can be performed to assess how a service is really perceived by the user, or how the Quality of Experience (QoE) is $[10,11]$.

Although a subjective evaluation of the QoE could be costly and time consuming, it gives more truthful results than an objective evaluation, which is merely based on QoS network performance parameters. QoE considers how users perceive and experience a multimedia communication service as a whole [29]. Since QoE relates to the user-perceived experience directly rather than to the implied impact of QoS, it is considered as a more important metric than QoS [30]. Tracking and predicting of users' satisfaction, service adaptation according to users' expectations, and maintain QoE at a satisfactory level are crucial for multimedia services, thereby leading to the development of reputation systems to manage subjective opinions in societies and yield a general scoring of a particular behavior [8].

Both from a theoretical and empirical perspective, the concept of QoE has been broadened over the last years. As a result, different definitions of QoE exist, but all have similar notion, referring to user satisfaction [31]. The ITU-T defined QoE as "the overall acceptability of an application or service, as perceived by the end-user", which might be influenced by 'user expectations' and 'context' [16]. Identifying, understanding, and quantifying the most determining aspects making or breaking the QoE of individual (or communities of) users and translating these rich insights into service and application optimization recommendations, is considered to be essential.

The objective of this paper is to identify device characteristics (platform), context parameters (day of the week, time of the day), and user aspects (familiarity with the service) that influence the user's usage behavior and QoE during VoIP calls. For the first time, the evolution of the user's usage behavior and QoE was investigated by analyzing the service usage and quality assessments of the VoIP service over a longer period of time. In addition, this paper quantifies the influence of the network and handovers during the call on the user's quality assessment. The results regarding the user's usage behavior are important in view of predicting the service usage and system load for mobile multimedia services. The analysis of the quality assessments can be used as a guidance to quantify (variations in) the QoE of mobile (VoIP) services. The results regarding the influence of the network and handovers can be used to optimize mobile data networks from a user point of view. The study of the evolution of the user's usage behavior and QoE over a longer period of time can be seen as a case study for long-term quality assessment of a service in a real environment.

\section{Related Work}

In many cases, the capabilities of VoIP technologies are analyzed using a network simulator [17] or in a private network [26], which enables the modification of the IP infrastructure and may have other characteristics regarding traffic or topology than the public Internet. Other experiments are performed in a predetermined environment covering a limited area, such as a university campus [6], thereby limiting the freedom of the test subjects with the risk of obtaining results that are not generally applicable. Moreover, the number of test subjects participating in a (mobile) QoE experiment is often limited to a few dozen due to time and budget constraints [11]. In contrast, this paper investigates the usage behavior and QoE during VoIP calls based on data of a large number of test subjects (more than thousand) making voice calls in their daily environment without any location, time or usage constraint. These test subjects are real customers of a commercial VoIP application, namely VikingTalk ${ }^{4}$, developed and managed by a Belgian mobile network operator, namely Mobile Vikings ${ }^{5}$. This eliminates any possible bias that is associated with the recruiting of test subjects who are asked to use a service merely for the sake of evaluation purposes.

To improve the quality in VoIP services, various approaches based on resource reservation have been proposed. Different call scheduling policies can be used when gateways with a limited capacity are used, thereby managing access to the network resources so that they are used in a fair manner [21]. The influence of QoS parameters, i.e., network related aspects, such as throughput, packet delay, or packet loss, on the QoE during VoIP calls has been studied extensively $[7,14]$. The de-

\footnotetext{
4 http://www.vikingtalk.com

5 http://www.mobilevikings.com
} 
gree of influence of QoS parameters on user perception has been determined and enabled the development of prediction models for multimedia services on mobile devices [2]. For VoIP calls, packet losses and the pattern of the packet losses, i.e., the distribution and duration of packet loss runs, has a significant influence on the perceptual quality of the conversation [3]. The wider the inter-loss gap duration, the lower is the quality degradation [18]. But also the jitter, i.e., the inter-packet delay encountered as packets are transmitted through the network from sender to receiver, has a significant influence on the quality of calls [4]. Especially for slower data networks such as UMTS (Universal Mobile Telecommunications System) or EDGE (Enhanced Data Rates for GSM Evolution) networks, these network related aspects have shown to be determining factors for the QoE during VoIP calls.

The new generation of cellular data networks, such as HSPA (High Speed Packet Access) and LTE (Long Term Evolution) networks, and the traditional WiFi networks offer a higher bandwidth satisfying the QoS targets [22] and reduce the risk of network impairments, such as packet loss and insufficient throughput, considerably. Moreover, forward error correction mechanisms using redundant data added to the voice stream can help to recover from these network impairments thereby sustaining audio quality [15]. As a result, QoS parameters do not always match with the user's QoE [6] during VoIP calls. The reduced risk of network impairments increases the relative impact of device characteristics, such as the platform, context parameters, such as the time, and user aspects, such as the familiarity with the service, during the use of a multimedia service. However, the influence of these device characteristics, context parameters, and user aspects on the user's usage behavior and QoE with a VoIP service has never been investigated according to our knowledge.

Moreover, traditional user experiments evaluate the QoE by taking a snapshot of the subjective experience of the user at one moment in time during the complete use process of the service [9]. The lack of any timerelated data in the analysis process implicitly denies much of the dynamic nature of the user's usage behavior [13] or experience with a service. Nevertheless, the user's behavior and experience continuously change over time, and are influenced by his or her prior expectations about the service [12]. Before people start using a particular product or service, they tend to already have some kind of preconception influencing their expectations [19]. After a user has adopted a product, and is using it more or less regularly, the actual use process evolves. As a user has more experience with the service, familiarity of the user increases, and this has an impact on how the service is being used. Karapanos et al. describe different phases a user goes through when using a product or service going from "the initial experiences with a service" over "giving the service a meaningful place in life" to "integrating the service in the user's lifestyle" [19]. Therefore, it is important that the user's usage behavior and $\mathrm{QoE}$ is not evaluated at a single point in time but rather over a continuous period during the use process. The resulting data can be understood as a time series of one feature, of which the clue for evaluation is the detection of trends in several successive time points [25]. This paper is the first to monitor trends in and analyze the evolution of the user's usage behavior and QoE with a VoIP service over a longer period of time (four months).

\section{VoIP Service}

Nowadays, a large variety of VoIP services is available. In this article, the user's usage behavior and experience with one of these services, namely VikingTalk, is investigated. VikingTalk offers users a multi-network VoIP telephony service, similar to the well-known Skype, enabling to call or receive calls from other VoIP users or people connected through the traditional fixed or mobile PSTN (Public Switched Telephone Network).

In contrast to Skype, the VikingTalk application has a transfer option that allows its customers to switch from VoIP (using the data connection of the available WiFi or cellular network) to the customer's primary mobile operator (using GSM to connect) during the same call. The mobile operator automatically takes over the VoIP call in case of a poor voice quality or loss of coverage on the data network, if users have enabled this handover process in the configuration settings. By a short beep sound during the voice call, users are informed about the transfer of the voice call from VoIP over the available data network to non-VoIP over the GSM network of the mobile operator. If a voice call initiated using VoIP was transferred to the GSM network of the mobile operator because of technical issues, it is not transferred again to VoIP (not even if the data connection is sufficiently recovered) in order to limit the possible disruptions introduced by the switching during the voice call.

In the configuration settings of the VikingTalk application, users can enable the handover process to switch automatically from a data network to the GSM network of the mobile operator. In addition, users have the possibility to influence this handover process. They can choose whether or not they want to use EDGE or 3G (3rd Generation) networks for their voice calls in case a WiFi connection is not available. For the VoIP service, 
a WiFi network is the first choice. If a WiFi network is not available, two alternative solutions exists for the voice call: either using the mobile data network (EDGE or $3 \mathrm{G}$ ) that is available through the user's mobile data plan (data credit) or using the GSM network of the user's mobile operator thereby charging the user as for a traditional phone call (voice credit). If the user opts not to use EDGE or $3 \mathrm{G}$, the voice call is transferred immediately to the GSM network of the mobile operator as soon as the user is out of range of the $\mathrm{WiFi}$ network. If the user opts to use EDGE or $3 \mathrm{G}$, this data network is preferred above the GSM network of the mobile operator. Then, the available EDGE or 3G network is used by default in the absence of WiFi, and only in exceptional cases when the throughput of the mobile data network (EDGE or 3G) becomes insufficient, the call is still switched to the GSM network of the mobile operator. The use of EDGE or 3G can induce a poorer voice quality compared to the GSM network of the mobile operator, but on the other hand, the use of $3 \mathrm{G}$ or EDGE can reduce the cost charged by the mobile operator for voice calls.

VoIP calls to another VoIP user are free for users of the VikingTalk application. VoIP calls to the fixed or mobile PSTN are charged based on the duration of the call. For voice calls that use the GSM network of the mobile operator (because of technical reasons), or voice calls initiated using VoIP that are transferred to the GSM network of the mobile operator, the rates offered by the mobile operator apply for the duration of the call over this GSM network. Charges for data traffic are not included in the VikingTalk rates and are charged separately.

For billing purposes and customer services, data about the VikingTalk service usage are internally stored and continuously monitored. Analysis of these data can provide insights into the parameters that influence the service usage and user's QoE. The analysis of this paper was based on a data set containing the details of all voice calls made by real customers of VikingTalk over a period of nearly four months (120 days), from October 1, 2012 to January 28, 2013. Since these customers were not aware of this study, they were not biased in any way. The resulting data set provides on the one hand a representative set of samples to investigate the influence of different parameters and on the other hand it allows to analyze trends in the user's usage behavior and QoE with the VoIP service over a longer period of time. The data set consists of objective, technical parameters regarding the call as well as subjective evaluations of the quality of the voice call.

The objective, technical parameters used to track the usage behavior are: an identification of the user who initiates the call and the user who receives the call, timestamps indicating the start and end of the call, the type of operating system of the user's phone, the duration of the voice call, the presence of handovers from data to GSM network, and configuration settings related to the handover process and the use of mobile data networks. After each voice call, the user has the opportunity to evaluate the quality of the VoIP service using a 5-point scale rating mechanism, thereby yielding a subjective evaluation of the user's experience with the VoIP service.

\section{Number of Calls}

The number of calls made by the users of the service is a measure that characterizes the usage behavior and is an indication of the popularity of the service.

\subsection{Characteristics of the Number of Calls}

The total number of calls, made by all users during the 120 -day period, is 127826 . So on average, 1065 calls are made each day. The standard deviation of the number of calls per day is 172.18 , indicating that the number of calls varies for subsequent days of the evaluation. During the evaluation period, 1050 subscribers of the VoIP service were active, each making on average 121.74 voice calls, or around 1 voice call per day. The users of the VikingTalk service are all smartphone users. Many of them are adolescents and students; but VikingTalk has customers with all kinds of backgrounds. So the users of VikingTalk are a representative set for the population of customers of a mobile operator.

\subsection{Differences in the Number of Calls per Platform}

During the evaluation period, 82300 calls or $64.4 \%$ were initiated on an Apple iPhone running iOS. The remaining 45526 calls or $35.6 \%$ are made using a mobile phone running the Android operating system. Also in terms of subscribers of the service, corresponding results are obtained. Of the 1050 subscribers who were active during the evaluation period, 622 users or $59.2 \%$ are iPhone users, and the remaining 428 users or $40.8 \%$ utilize an Android device.

So although the popularity of (new) Android devices at the end of 2012 and the beginning of 2013 [23], the majority of the voice calls are made on iOS. During the evaluation period, the VikingTalk application was not (yet) available for other mobile platforms such as Blackberry or Windows Mobile. 


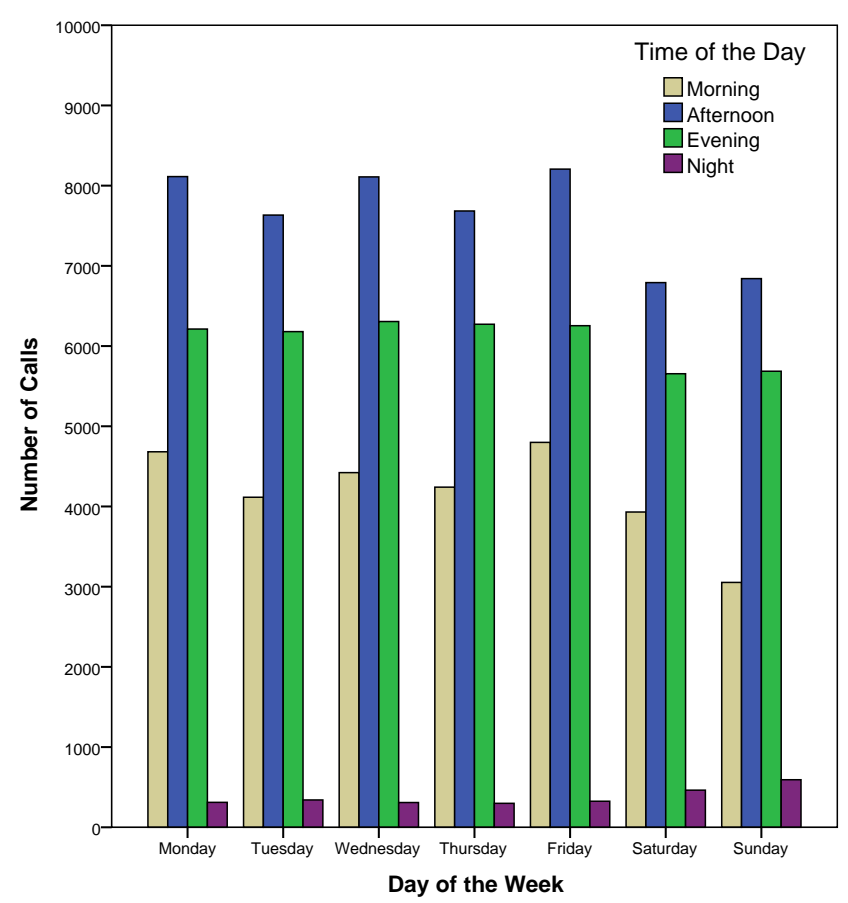

Fig. 1 Histogram of the number of calls depending on the day of the week and time of the day

\subsection{Influence of the Day of the Week on the Number of Calls}

Figure 1 shows the total number of calls, made by all users of the VoIP service, partitioned by the day of the week. During weekdays, the registered number of calls is ranging from 18269 (for calls on Tuesdays) to 19584 (for calls on Fridays) with an average number of 18963 calls per weekday. Since the evaluation period consisted of 17 weeks, this amounts to an average of 1115 calls per day on weekdays. In contrast, the total number of calls made on Saturdays is 16840, and on Sundays, 16173 calls are made during the evaluation period. So on average, 971 calls are made per day on a weekend day, or $12.9 \%$ less calls than on weekdays. The significantly higher number of calls made on weekdays compared to weekend days indicates that users are utilizing the VoIP service more often on weekdays than on weekend days.

\subsection{Influence of the Time of the Day on the Number of} Calls

A more detailed analysis of the service usage can be made by partitioning the calls according to the time of the day. For this analysis, a day is split into four periods of six hours: morning, from $6 \mathrm{AM}$ to $12 \mathrm{PM}$, afternoon, from $12 \mathrm{PM}$ to $6 \mathrm{PM}$, evening, from $6 \mathrm{PM}$ to $12 \mathrm{AM}$, and night, from $12 \mathrm{AM}$ to $6 \mathrm{AM}$.
Figure 1 indicates that significant differences in the user activity exist between the different time periods. The afternoon is the most popular moment to make voice calls, with a total of 53377 calls (or $41.7 \%$ ). The second most popular time is the evening, with 42565 calls (or $33.3 \%$ ) in total, followed by the morning, during which 29243 calls (or $22.9 \%$ ) were made. Not surprisingly, the least voice calls were made during the night (2641 calls or $2.1 \%$ ).

In general, less voice calls are made on Saturdays and Sundays compared to weekdays, at least in the morning, afternoon, and evening. In contrast, during night more calls are made on Saturdays (463) and Sundays (593) than on weekdays (317 on average). Over the evaluation period of 17 weeks, on average 31 voice calls are made during each weekend night, whereas the average number of voice calls during a night in the week is only 19. This significant difference can be explained by the difference in users' life style during weekend and weekdays.

4.5 Influence of the Hour of the Day on the Number of Calls

The variability in the activity of the users during the day can be further investigated by partitioning the calls by the hour in which they are made. Figure 2 shows the total number of calls, made by all users of the VoIP service, partitioned by the hour of the day.

A clear usage pattern is visible corresponding to most people's way of life. A very low activity is witnessed during the middle of the night (2 AM to $5 \mathrm{AM}$ ). As the early birds get up (5 AM to $8 \mathrm{AM})$, more voice calls are made. The number of voice calls continues to increase, as more people start their day (8 AM to 11 $\mathrm{AM})$. Then, the number of voice calls per hour remains stable till after lunch time (11 AM to $3 \mathrm{PM})$. During the afternoon (3 PM to $7 \mathrm{PM})$, the number of voice calls increases until reaching the peak in activity around 6 PM. During the first hours of the evening (7 PM to $9 \mathrm{PM})$, still a lot of voice calls are made. Later in the evening (9 PM to $1 \mathrm{AM}$ ), as people go to bed, the number of voice calls per hour decreases. This decrease in activity continues during the first hours of the night (1 AM to $2 \mathrm{AM}$ ).

Noteworthy is the difference between the peak hour in which most calls are made (10189 calls from 6 PM to $7 \mathrm{PM}$ ) and the hour in which the fewest calls are made (only 162 calls from $3 \mathrm{AM}$ to $4 \mathrm{AM}$, or $1.6 \%$ of the calls during the peak hour). This pattern in the usage behavior can be used to predict the system load in view of reserving network resources. 


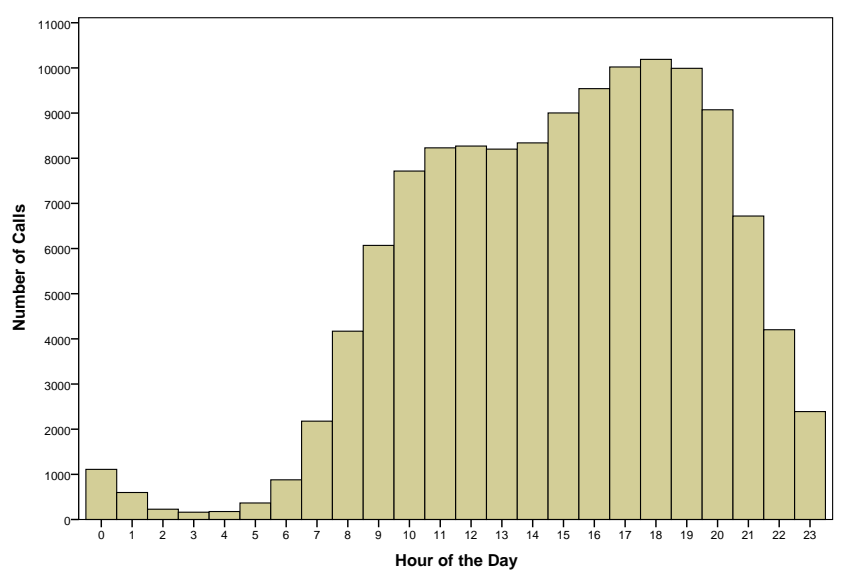

Fig. 2 Histogram of the number of calls depending on the hour of the day

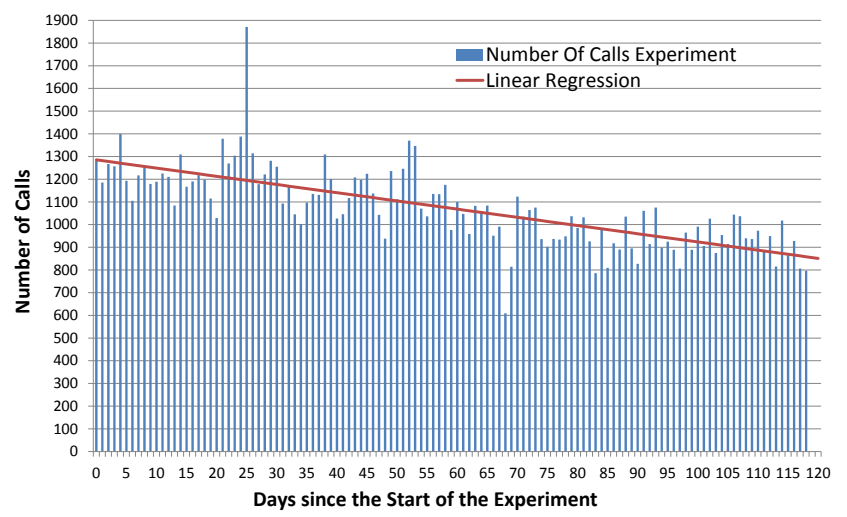

Fig. 3 The evolution of the number of made voice calls over time

\subsection{Evolution of the Number of Calls over Time}

As users become more familiar with the service, their usage behavior and perceptions of the service quality may change over time. Therefore, evaluating the usage of a service over a longer period of time is essential to quantify the user's usage behavior and experience with a service. Such an evaluation outside the constraints of a classical user test, over a long period of time, has never been performed up to now for a widely-used, mobile, multimedia service, such as the VikingTalk VoIP service.

The evolution of the usage of the service over time is analyzed by monitoring the number of calls day by day. Figure 3 visualizes the number of calls, made by all users of the service, per day since the start of the evaluation. As mentioned in Section 4.1, the average number of calls per day over the complete evaluation period is 1065. The graph shows a large variability of the number of calls each day (standard deviation $=172.18$ ). Moreover, a slight decrease in the number of the voice calls per day can be denoted over the evaluation period.
This decrease is further investigated by a regression analysis, a statistical process for estimating the relationships between a dependent variable and one or more independent variables. The independent variable is the sequence number of the day since the start of the evaluation. The dependent variable is the number of calls made by all users of the service on that specific day.

Equation (1) shows the result of the linear regression, which is also visualized in Figure 3 by the red line. The slope of this model is significant $(p<0.005)$, indicating a significant evolution of the number of calls over time. The model has an $R^{2}$ value of $0.5195 . R^{2}$ is the coefficient of determination and represents the proportion of variability in the data set that is accounted for by the statistical model.

NumberOfCalls $=1285.1761-3.6167 \cdot$ Days SinceStartExperiment $(1)$

\section{Call Duration}

Just as the number of calls, the call duration is also an interesting characteristic of the usage behavior of users of the service. The call duration is measured as the time between the moment when the person being called answers the call (or voice mail starts recording) and the time when one of the two persons hangs up the phone.

The voice calls which are not answered, and as a result have a duration of 0 seconds, are not taken into account in this analysis. During the evaluation period, 20438 voice calls or $15.9 \%$ were not answered.

\subsection{Characteristics of the Call Duration}

The average call duration for the voice calls made during the evaluation period is 270 seconds or 4.5 minutes. But large differences in the call duration exist depending on the context (day of the week, hour of the day, etc.), thereby inducing a large variability in the duration (standard deviation of 537 seconds).

Figure 4 shows the histogram of the call duration. The duration of the call is shown on the horizontal axis in intervals of 60 seconds; the number of calls with such a duration is visible on the vertical axis. To prevent misuse of the service, all voice calls are automatically disconnected after one hour. As a result, all calls have a duration shorter than 3600 seconds.

As shown in Figure 4, most voice calls have a very short duration (shorter than 1 minute). The number of calls with a longer duration decreases as the duration increases. More specifically, the number of calls is found to vary as a Pareto distribution of the duration of 


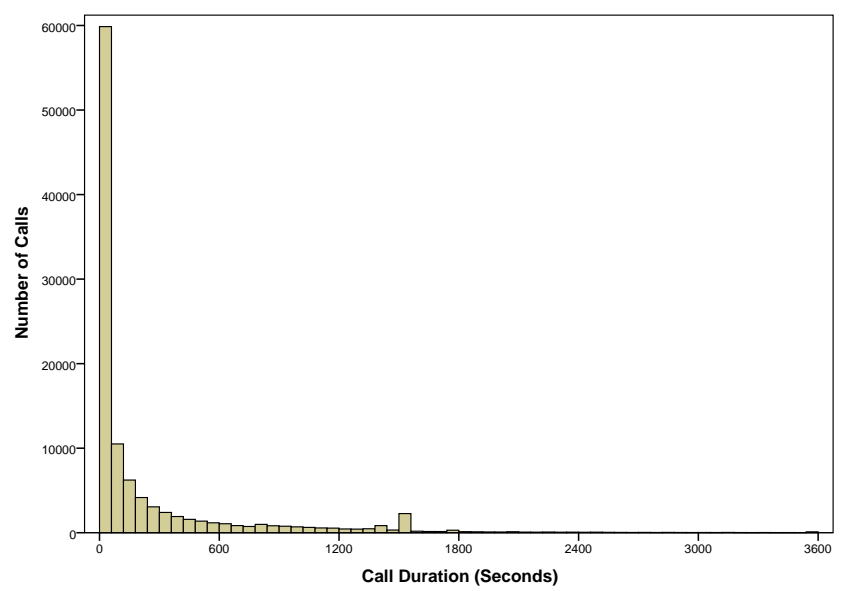

Fig. 4 Histogram of the call duration

the call. The Pareto distribution, is a power law probability distribution that coincides with social, scientific, geophysical, actuarial, and many other types of observable phenomena [27]. Predicting the call duration can be interesting for QoS applications, in which resources have to be reserved during the voice call.

\subsection{Differences in Call Duration per Platform}

Comparison of the call duration for the different mobile platforms reveals that calls made on Android last approximately 20 seconds longer (mean duration 281.90 seconds) than calls made on iOS (mean duration 261.65 seconds). This difference is significant according to a $\mathrm{T}$ test in which the following parameters were obtained: degrees of freedom $=105871.24, \mathrm{t}=6.29, p<0.005$. So, although most VoIP calls are made on iOS (Section 4.2), the calls on iOS have typically a shorter duration than the calls made on Android phones. A possible reason for this might be the fact that Android and iOS have different kinds of users, e.g., different age groups.

\subsection{Influence of the Day of the Week on the Call Duration}

Figure 5 shows the mean duration of the voice calls, as well as the $95 \%$ confidence interval of the mean duration, depending on the day of the week. This graph is based on the data of the calls made during the 120-day evaluation periode by all users of the service.

Limited differences in the mean call duration are visible. Noteworthy is the mean call duration on Sundays (295.12 seconds) which is significantly higher then the mean call duration on other days (266.69 seconds). This difference of approximately 28 seconds can be ex-

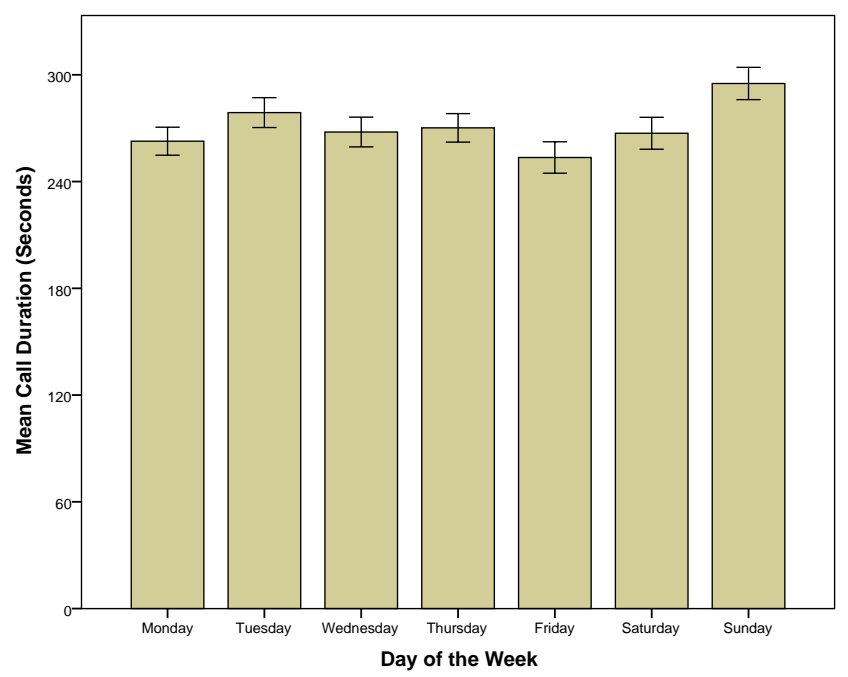

Fig. 5 The mean duration of the call depending on the day of the week

plained by the additional leisure time and the typically less-stressful life style of people on Sundays.

\subsection{Influence of the Time of the Day on the Call Duration}

The call duration is further investigated by making a distinction based on the time of the day. Figure 6 shows the mean call duration, as well as the $95 \%$ confidence interval of the mean duration, depending on the day of the week and the time of the day. In the morning and afternoon, users have generally short conversations (mean call duration of respectively 202.71 and 213.29 seconds). These calls are typically work-related or regarding practical arrangements and therefore concise. In contrast, calls during the evening and night have a considerably longer duration (mean call duration of respectively 375.26 and 405.28 seconds). So, calls during the evening and night are approximately $90 \%$ longer than the calls made during the morning and afternoon. Evening and night calls are typically not work-related, regarding the user's personal life and so more verbose. In addition, the rates charged by many telecommunications service providers are lower during the evening and night compared to the morning and afternoon. Therefore, users might be inclined to make longer voice calls during the evening and night.

Also the standard deviation of the duration is larger for calls made during the evening and night (respectively 620.12 and 664.67 seconds) compared to calls at other times. In contrast, the short mean duration of the calls during the morning and afternoon is coupled with a small standard deviation (respectively 489.66 and 460.99 seconds). 


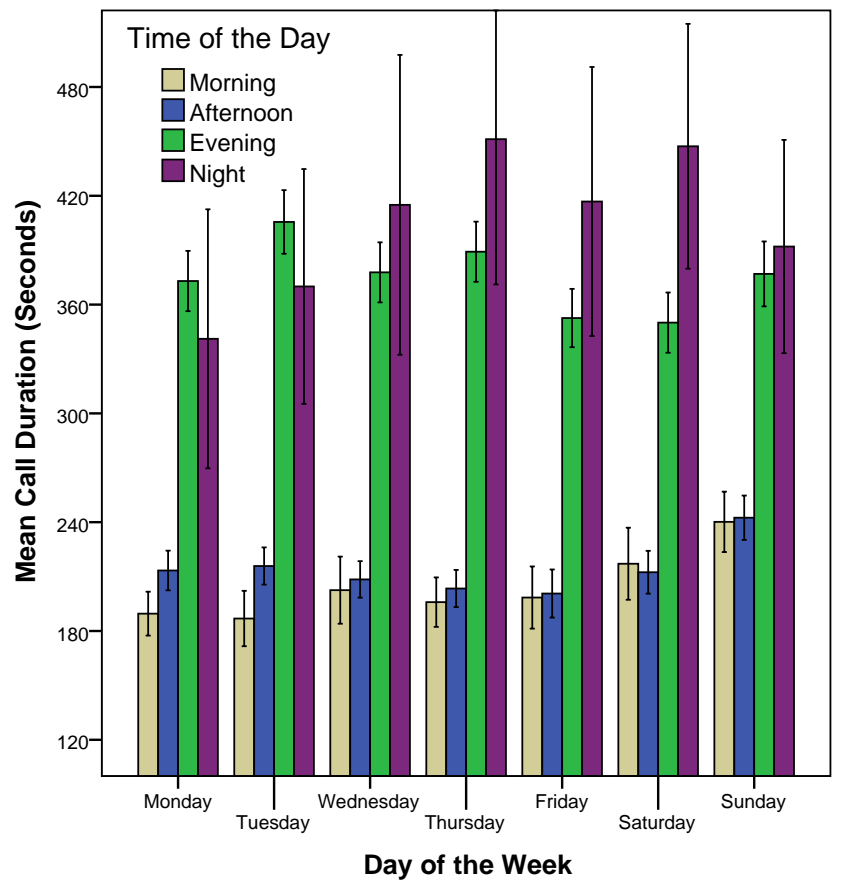

Fig. 6 The mean duration of the call depending on the day of the week and the time of the day

Also noteworthy is the significantly longer duration of calls on Saturday nights (mean duration $=447.29$ ), compared to the other times of the day on Saturdays (mean duration $=259.84)$. This longer duration can be explained by the nightlife activities of people on Saturday nights. This difference in duration is less remarkable or not significant on other days of the week.

\subsection{Influence of the Hour of the Day on the Call Duration}

The variability of the call duration during the day can be further investigated by analyzing the duration depending on the hour during which the calls were made. Figure 7 shows the mean call duration, as well as the $95 \%$ confidence interval of the mean duration, for each hour of the day.

As in Figure 2 showing the number of calls per hour, Figure 7 reveals a pattern of the call duration corresponding to the life style of a typical user. The voice calls with the shortest mean duration are the ones during the early hours of the day (5 AM to 7 AM). During the morning and afternoon (7 AM to $5 \mathrm{PM}$ ) the call duration is quite short (mean duration $=205.70$ seconds) and remains approximately constant over the time. During the day, users generally keep the conversations short as they are busy with their daily activities. After the business hours, as more people come home

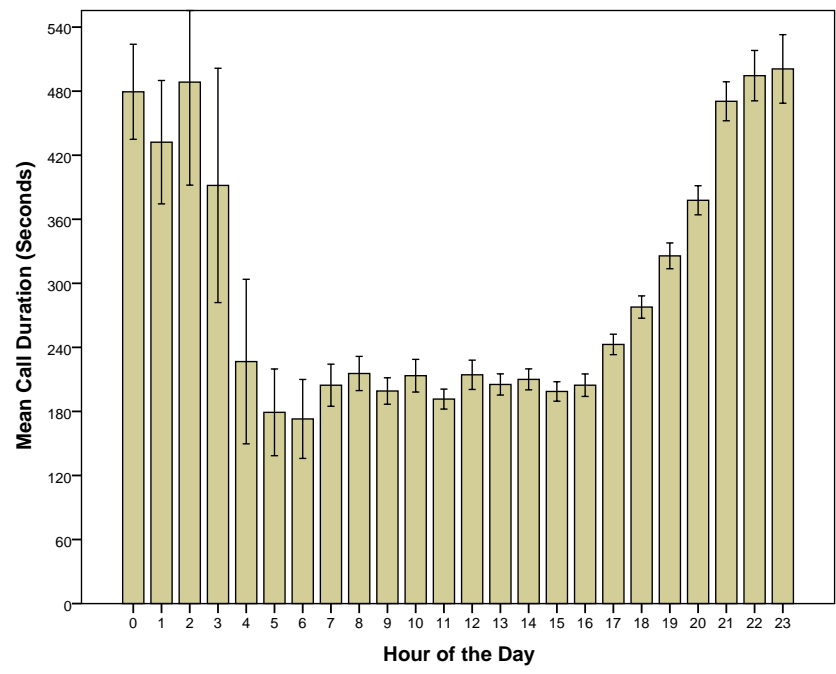

Fig. 7 The mean duration of the call depending on the hour of the day

(5 PM to $11 \mathrm{PM}$ ), the mean call duration increases. At this moment of the day, people have generally more time to call their friends or family and make long voice calls. The peak in mean call duration (500.84 seconds) is reached later on during the evening (11 PM to 12 AM). During the first hours of the night (12 AM to 3 $\mathrm{AM}$ ) the mean duration of the calls is still quite long (466.68 seconds). Later on during the night (3 AM to 5 $\mathrm{AM})$ the mean call duration decreases as more people are asleep and only urgent and concise voice calls are made.

\subsection{Evolution of the Call Duration over Time}

By monitoring the mean duration of the voice calls day by day, the usage behavior of the users can be further analyzed. Figure 8 shows the mean duration of the calls, made by all users of the service, per day since the start of the evaluation. Over the subsequent days, the variability in mean call duration is limited (standard deviation $=28.23$ seconds).

Analyzing the complete evaluation period reveals a slight increase of the mean call duration over the 120 days. By means of a regression analysis, the increased call duration is further investigated and quantified. The independent variable is the sequence number of the day since the start of the evaluation. The dependent variable is the mean duration of the calls made by all users of the service on that specific day.

The result of this linear regression is shown in Equation (2) and visualized in Figure 8 by the red line. The slope of this model is significant $(p<0.005)$, thereby 


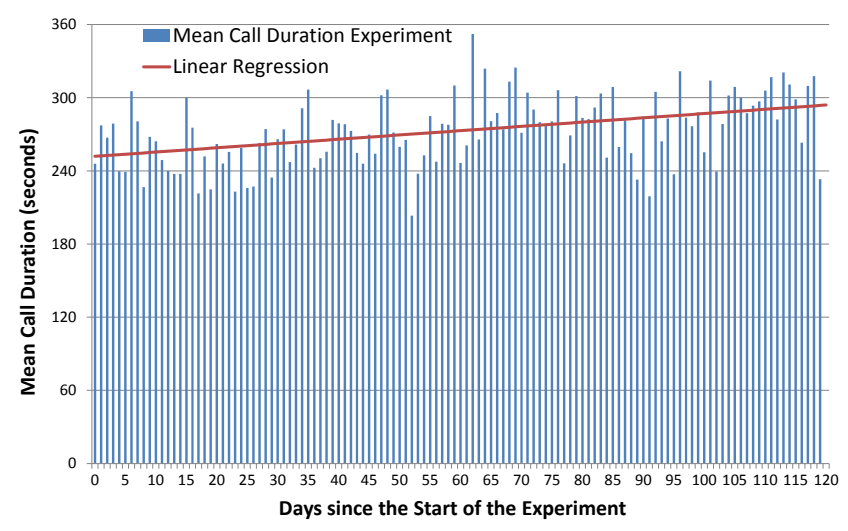

Fig. 8 The evolution of the mean call duration over time

indicating a significant evolution of the call duration over time. The $R^{2}$ value of the model is 0.1868 .

Duration $=251.9015+0.3507 \cdot$ DaysSinceStartExperiment

Although the decreasing number of calls over the evaluation period (Section 4.6) might give the impression of a decrease in popularity of the service, the increase of the mean call duration contradicts this. So over time, users are making less voice calls but the mean duration of these voice calls is becoming longer. This indicates that the VoIP service is becoming more mature. Over the time, users are utilizing the VoIP service less as a nice-to-have gadget to experiment and make short calls. Rather, they are starting to consider the VoIP service as a valuable alternative for the traditional GSM network of their mobile operator to make longer calls.

\section{Characteristics of the Quality Rating}

The quality rating, as expressed by the user immediately after the voice call, reflects the quality of the service as experienced by the user and can be used as an estimation of the user's QoE with the service. Since providing a quality rating is an optional feature that users can disable in the application, only 30384 of the voice calls, or $23.8 \%$ of the calls, are evaluated by the users on the 5-point scale rating mechanism.

\subsection{Typical Quality Rating}

For the calls made during the 120-day evaluation period, the mean rating is 3.15 with a standard deviation of 1.66. Figure 9 shows that the distribution of the ratings for the voice calls is not uniform. This histogram indicates that users tend to provide 'extreme' ratings, 1 or 5 , rather than moderate ratings, 2,3 , or 4 . This is a typical user behavior for 5 -point scale mechanisms, and was already witnessed for ratings on the YouTube website [28].

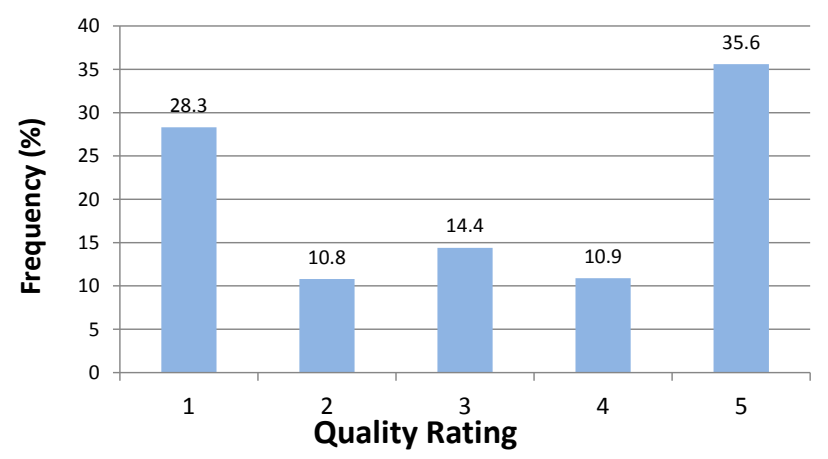

Fig. 9 The distribution of the subjective ratings of the users evaluating the voice calls

\subsection{Differences in the Quality Rating per Platform}

During the evaluation period, 22363 voice calls made on an iPhone running iOS are evaluated by the users, whereas the number of evaluated calls on Android devices is 8021 . The mean rating on $\mathrm{iOS}$ is 3.23 . For Android, the mean rating obtained during the evaluation period is 2.91. This difference of 0.32 is significant according to a T-test with the following resulting parameters: degrees of freedom $=15465.22, \mathrm{t}=-15.31, p<$ 0.005. As a result, the experience of the users with the VoIP service is better on iOS than on the Android platform. This difference in experienced quality can be explained by the difference between the high-end iPhones, well capable to run the VoIP application smoothly, and the many low-end Android phones, which might induce a lower user experience due to limited resources in terms of memory and processing power. Analyzing the specifications of the phones used during the experiment showed a positive correlation between the phone capabilities and the users' quality assessments.

\subsection{Influence of the Day of the Week on the Quality Rating}

Figure 10 shows the mean quality rating of the voice calls, as well as the $95 \%$ confidence interval of the mean quality rating, depending on the day of the week. This graph is based on the data of the calls made during the 120-day evaluation periode by all users of the service. Limited differences in the mean quality rating are visible between the different days of the week.

An ANOVA (ANalysis Of VAriance) is used to determine whether or not the means of several groups are all equal, and therefore generalizes the T-test to more than two groups [20]. An ANOVA hypothesis test showed that no significant difference $(p=0.26)$ in quality rating exists between the different days of the week. The null-hypothesis, $H_{0}$, which assumes that the mean 


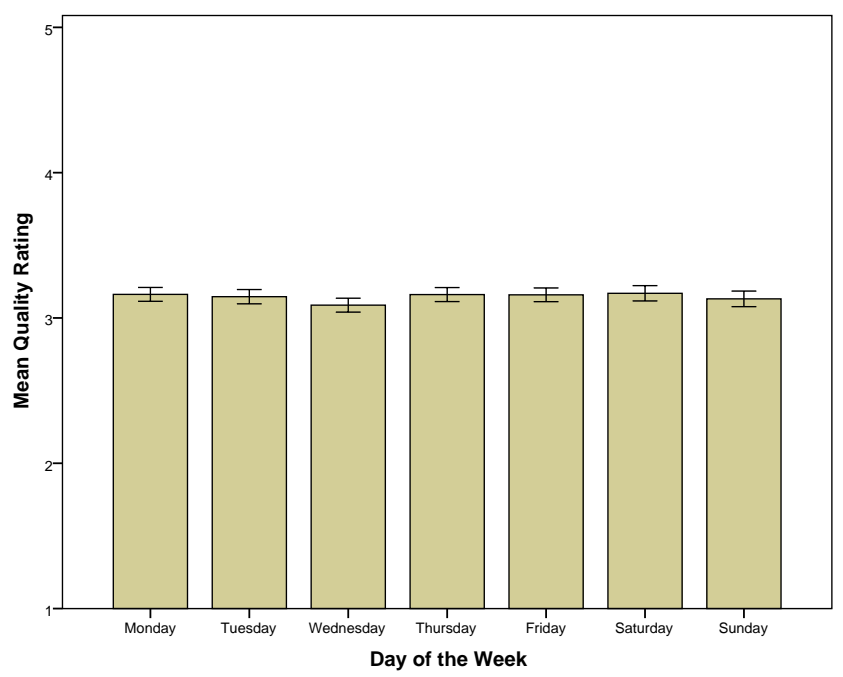

Fig. 10 The mean quality rating of the call depending on the day of the week

quality rating is the same for every day of the week, is not rejected.

6.4 Influence of the Time of the Day on the Quality Rating

The differences in mean quality rating are further investigated by making a distinction based on the time of the day. Figure 11 shows the mean quality rating, as well as the $95 \%$ confidence interval of the mean quality rating, depending on the day of the week and the time of the day. The results indicate that the mean quality rating is lower for calls made during the night, compared to the mean quality rating of calls during daytime. Especially on Mondays, this difference in mean quality rating is significant. During the weekend, the difference in quality rating between day and night is smaller.

Because of the limited user activity with the service and the low network load during the night (Figure 2), the technical conditions are optimal for a VoIP call. Therefore, in a purely-objective assessment of the VoIP service, a higher quality rating would be expected during the night. However, because the user rating is a subjective assessment that is influenced by user aspects such as the context and the mood of the user, a lower rating is obtained for calls during the night. Different aspects might have a negative influence on the user's subjective rating for calls during the night, e.g., tiredness or frustration due to be awake, bad news via the night call, inappropriate timing of the call, etc. The real reason why users provide a lower quality assessment during the night is an interesting topic for future research. No significant differences are identified between

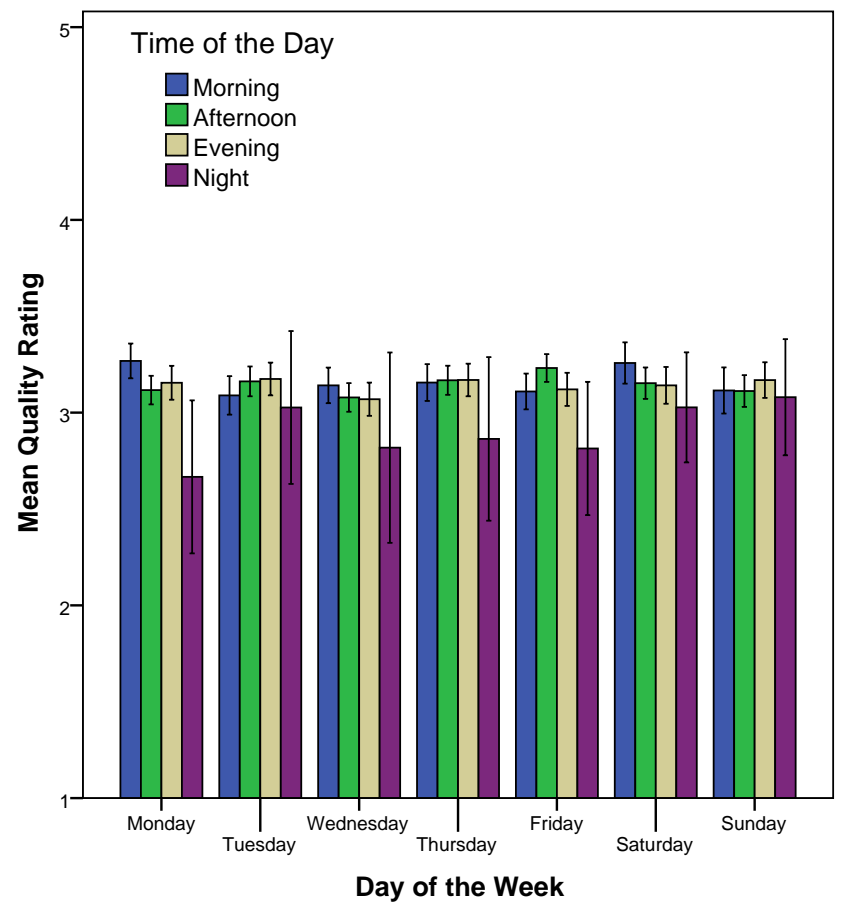

Fig. 11 The mean quality rating of the call depending on the day of the week and the time of the day

the mean quality rating for calls during the morning, afternoon, and evening.

\subsection{Influence of the Hour of the Day on the Quality} Rating

The differences in mean quality rating on different times of the day are further investigated by analyzing the mean rating depending on the hour during which the calls were made. Figure 12 shows the mean quality rating, as well as the $95 \%$ confidence interval of the mean quality rating, for each hour of the day.

The graph of Figure 12 shows that significant differences in quality rating exist for different hours of the day. During the day and the early hours of the evening (9 AM to $10 \mathrm{PM}$ ), the mean quality rating only slightly varies, with a score ranging between 3.02 and 3.32. Small differences are visible for subsequent hours, for example the difference between 3 PM (3.21) and 4 PM (3.05) might be due to the end of school time. Noteworthy is the low mean rating during the night (3 AM to 6 $\mathrm{AM}$ ), significantly lower than during the day. Because of the low number of calls during the night, the confidence intervals are larger during the night than during the day (Figure 11 and 12). The lowest mean quality rating (2.47) is obtained during night at $5 \mathrm{AM}$, whereas the highest mean rating (3.46) is obtained during the evening at $10 \mathrm{PM}$. This difference of approximately one 


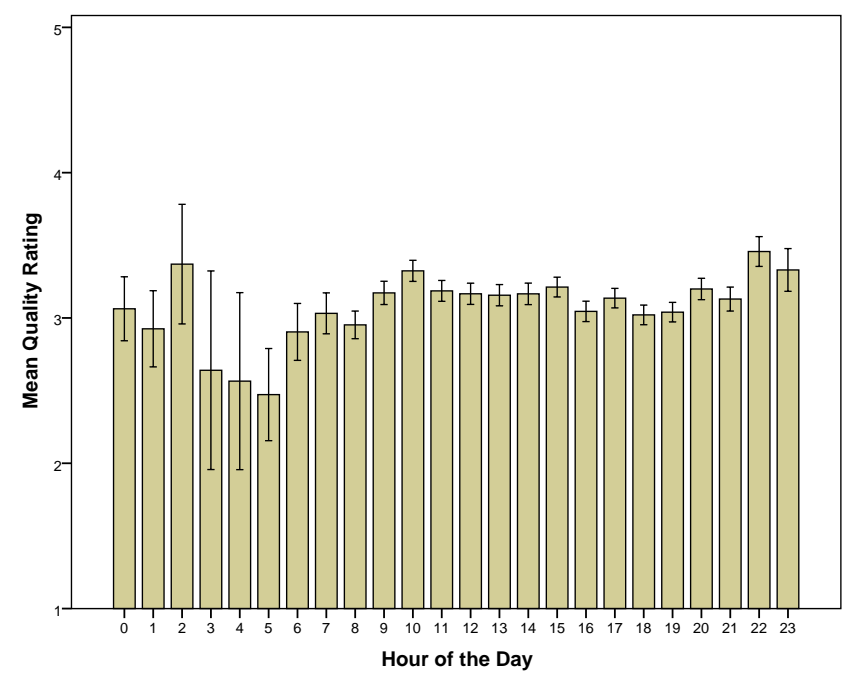

Fig. 12 The mean quality rating of the call depending on the hour of the day

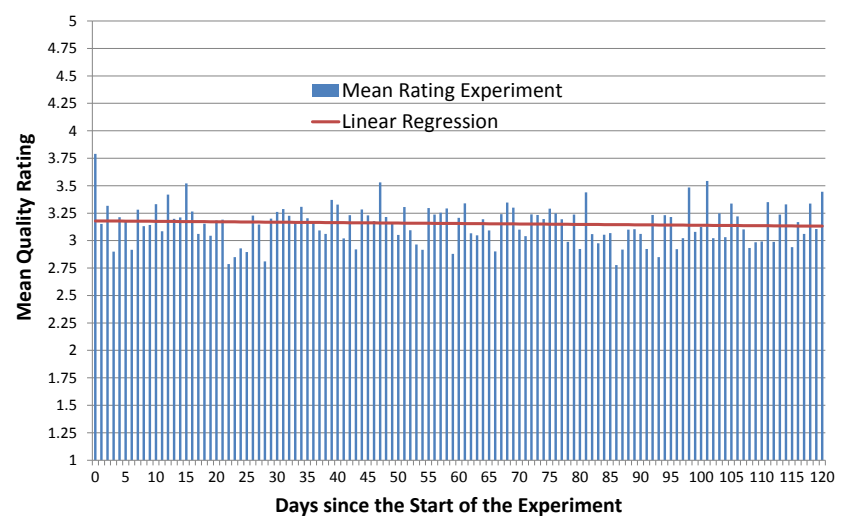

Fig. 13 The evolution of the mean quality rating over time

unit on a 5-point scale rating mechanism demonstrates the influence of the context and the mood of the user during subjective quality assessments.

\subsection{Evolution of the Quality Rating over Time}

The subjectively-perceived quality of the VoIP service is further analyzed by monitoring the mean quality rating of the voice calls day by day. Figure 13 shows the mean rating of the calls, made by all users of the service, per day since the start of the evaluation. The graph shows that the mean quality rating, calculated for each day of the evaluation period, varies only slightly (standard deviation $=0.17$ ).

The evolution of the mean quality rating over time is investigated by means of a regression analysis. The independent variable is the sequence number of the day since the start of the evaluation. The dependent variable is the mean quality rating of the calls made by all users of the service on that specific day.
The result of this linear regression is shown in Equation (3) and visualized in Figure 13 by the red line. However, the slope of this model showed not to be significantly different from zero $(p=0.33)$, thereby indicating that the quality rating does not increase or decrease significantly over time. The $R^{2}$ value of the model is 0.0080 .

MeanQualityRating $=3.1796+0.0004 \cdot$ Days SinceStartExperiment $(3)$

\subsection{Evolution of the Quality Rating over Service Usage}

As users become more familiar with the service, their perceptions of the service quality may change over time. However, no trend in the mean rating over the subsequent days of the evaluation period is visible in Figure 13. The absence of a trend over time can be explained by the different usage patterns of the users. On average, users make one voice call a day. But many users utilize the service only sporadically; and some users utilize the service very intensively (up to 22 calls a day). As a result, different users get familiar with the service and gain experience with the usage of it at a different rate. Users who utilize the service very intensively are familiar with it after a few days. In contrast, users who utilize the service sporadicly need more time to get familiar with it, and their experience with the service will evolve slower.

Therefore, the evolution of the quality ratings can be better investigated as a function of the user's usage and familiarity with the service. Figure 14 shows the mean quality rating over the subsequent voice calls made by the user. The number of calls made by the user can be seen as an indication of the user's familiarity with the service. During the evaluation period, 257 users or $24.5 \%$ made 120 or more calls. The intensive usage of the service ensures that these users get more experience with the service and are becoming more familiar with it.

The graph clearly shows a decrease of the mean quality rating over the first 120 voice calls made by the users. This is confirmed by a linear regression analysis that has as the independent variable the number of calls already made by the user, and as dependent variable the mean quality rating at that moment.

The resulting linear model has a significant slope for the number of calls made by the user $(p<0.005)$, thereby indicating that the mean quality rating decreases significantly as the familiarity with the service increases. The $R^{2}$ value of the model is 0.2885 . Equation (4) shows that the mean quality rating is 3.22 for the first call of the users and that this mean quality rating slightly decreases as users have utilized the ser- 


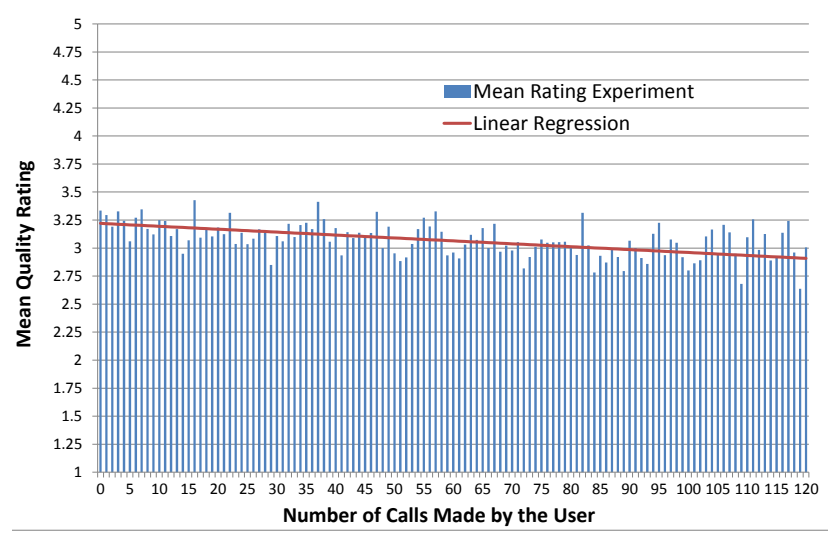

Fig. 14 The evolution of the mean quality rating over service usage

vice more often. After making 120 voice calls, the mean quality rating is 0.31 lower compared to the first call.

MeanQualityRating $=3.2229-0.0026 *$ NumberOfCallsMade(4)

This decrease in mean quality rating can be explained by the evolution in the user's familiarity and experience with the service. When users start utilizing a service, they have some expectations influencing their judgment of the service. When users utilize the service more often, they become more experienced and familiar with the service, thereby adjusting their expectations. Familiarity with the service might induce higher expectations, based on the users' previous experiences with the service. Higher expectations may in turn lead to a lower quality assessment. In addition, after using the service several times, users might pay more attention to the quality, thereby noticing more artifacts in the audio, and as a result perceiving the quality of the service differently. Important to note is that this trend does not continue during extended use of the service. For intensive users, we notice that the subjective quality stagnates after about 120 calls; users are fully familiar with the service and the mean rating remains constant.

\section{Network and Handover Influences}

In order to quantify the user's quality rating and in the end improving the QoE, analyzing the influence of the different technical parameters is of vital importance. This section discusses in detail the influence of the network that was used and handovers during the voice call. Figure 15 summarizes the effect of the considered parameters on the subjective quality rating of the user. As explained in Section 3, users can enable or disable the handover process in the configuration settings of the application. Users can opt to enable this handover process in order to make voice calls possible in case the data connection is lost or opt to disable this handover process to reduce the cost charged by their mobile operator. Analyzing the data set shows that the automatic handover process was enabled for $10.0 \%$ of the evaluated calls, or 3045 calls.

If the handover process is disabled, the call is under no circumstances transferred to the GSM network of the user's mobile operator. In this case, the VikingTalk application attempts to initiate the call over an available WiFi network. If a WiFi network is not available, the voice call can be made using a connection over a cellular data network (EDGE or 3G). In order to make this possible, the user has to enable the option to use EDGE or $3 \mathrm{G}$ for voice calls in the configuration of the application. For approximately $23.8 \%$ of the voice calls, users have enabled the use of EDGE or 3G. Figure 15 demonstrates that for voice calls during which no handover occurs, the calls made over a WiFi network receive a higher mean quality rating, than the calls in which the option for EDGE or $3 \mathrm{G}$ is enabled. The difference of 0.34 showed to be significant according to a statistical T-test with the following resulting parameters: degrees of freedom $=13333.69, \mathrm{t}=16.33, p<0.005$.

Even if the handover process is enabled, many voice calls will be made without a handover. Only in case of an insufficient throughput or loss of coverage on the data network, the VikingTalk service falls back on the network of the user's mobile operator. For most voice calls, network conditions are stable and such handover is not necessary. In about $1.1 \%$ of the cases, or 336 calls with a rating, an handover from a data- to GSMnetwork occurred. Because such a data-to-GSM handover can introduce distortions during the call, calls during which such an handover occurs receive generally a lower rating from the user than calls without handovers. Figure 15 indicates that the mean difference between a call without and a call with handover is 0.14 if only WiFi is used, and 0.09 if EDGE/3G networks are enabled. Since the voice quality obtained by using EDGE or $3 \mathrm{G}$ is typically lower than the quality over $\mathrm{WiFi}$, the additional distortion introduced by a handover has a lower impact if the VoIP call is using an EDGE or $3 \mathrm{G}$ network.

Also for calls during which a data-to-GSM handover occurs, a significant difference in quality rating is witnessed between calls in which EDGE/3G networks are enabled and calls that use only WiFi networks (difference of 0.29 ). As a result, the use of EDGE or 3G networks and the occurrence of data-to-GSM handovers during the voice call have a significant negative impact on the subjectively-perceived quality of the call. 


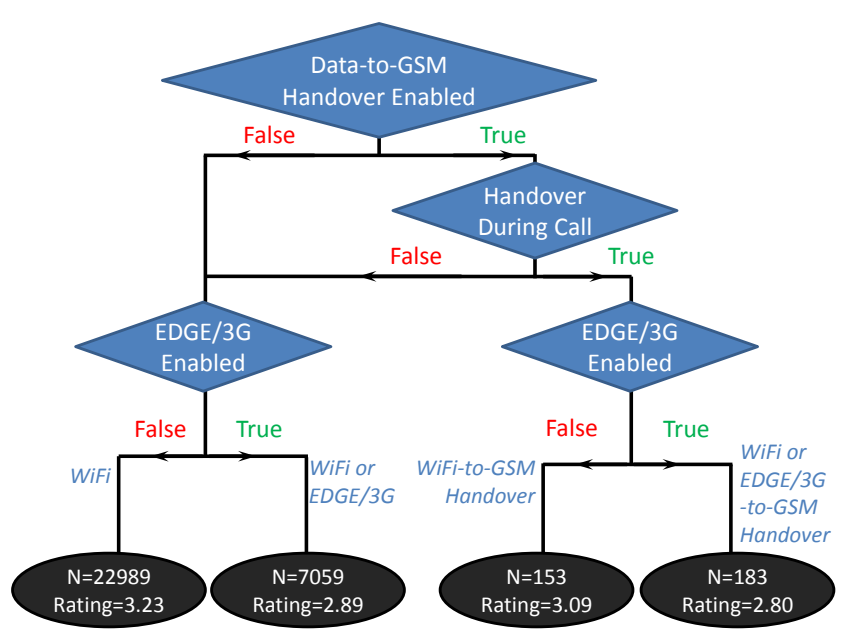

Fig. 15 The decision diagram showing the mean rating that was obtained depending on the used network and a possible handover.

\section{Conclusions}

In this paper, the user's usage behavior and QoE with a commercial VoIP service is investigated by analyzing the service usage and subjective quality assessments of more than thousand actual users of the service in their daily environment without any restrictions.

The paper shows the influence of device characteristics, such as the platform, context parameters, such as the time, and user aspects, such as the familiarity with the service, on the number of calls made by the users, the duration of the calls, and the users' quality ratings for the calls. In contrast to traditional studies on the usage behavior and QoE, this analysis is not based on a short evaluation at one specific moment in time; but users are monitored during an evaluation period of 120 days in order to investigate the evolution in usage behavior and QoE during service usage.

Regarding the number of calls, the VoIP service is most popular among iPhone users. Compared to weekdays, less calls are made during the weekend. The evening is the most popular time for making voice calls, with a peak in the number of calls around 6 PM. Over the 120days evaluation period, the number of calls decreases slightly.

In terms of call duration, iPhone users made calls that are slightly shorter than the calls of Android users. The longest calls are typically made on Sundays, when users have more leisure time. During the morning and afternoon the calls are significantly shorter than during the evening and night. The peak in call duration is reached around $11 \mathrm{PM}$. In contrast to the number of calls, the call duration increases over the 120-days evaluation period, thereby indicating that the user's usage behavior with the service is evolving over time.
The user's experience with (the quality of) the service is assessed by the user's subjective ratings. Due to many low-budget phones running Android, the ratings of Android users are in general slightly lower than the ratings of iPhone users. For calls during the night, lower quality ratings are obtained than during the day. Since these ratings are subjective assessments of the user, non-technical aspects such as the context and the mood of the user have an influence on the evaluation procedure. Provided that there is a strong correlation between time of day and the mood of users (which there is reason to believe), the study indicates that the user's mood is a significant parameter of the QoE. Also other parameters, such as the location of the user or the content of the call, might have a significant influence on the QoE. Investigating these contextual aspects constitutes interesting opportunities for future research.

As users have utilized the service more often and became more familiar with it, the perceived quality showed to decrease slightly over the first 120 calls. These results prove that the usage behavior and QoE can evolve during the entire use process of the service due to adjusted expectations, previous experiences with the service, and a change in the user's intentions. Regarding the technical parameters of the call, the results showed that the use of EDGE or 3G networks and the occurrence of data-to-GSM handovers during the voice call have a significant negative impact on the perceived quality of the call.

\section{References}

1. About.com: What Affects Voice Quality in VoIP Calls (2013). Available at http://voip.about.com/od/ voipbasics/a/factorsquality.htm Accessed on 5 July 2013.

2. Agboma, F., Liotta, A.: Quality of experience management in mobile content delivery systems. Telecommunication Systems 49(1), 85-98 (2012). DOI 10.1007/ s11235-010-9355-6. URL http://dx.doi.org/10.1007/ s11235-010-9355-6

3. Alvarez-Flores, E., Ramos-Munoz, J., Ameigeiras, P., Lopez-Soler, J.: Selective packet dropping for voip and tcp flows. Telecommunication Systems 46(1), 1-16 (2011). DOI 10.1007/s11235-009-9252-z. URL http: //dx.doi.org/10.1007/s11235-009-9252-z

4. Benaissa, M., Lecuire, V., Lepage, F., Schaff, A.: Efficient de-jitter control for voice applications over wireless ad hoc networks. Telecommunication Systems 28(2), 211230 (2005). DOI 10.1007/s11235-004-5017-x. URL http: //dx.doi.org/10.1007/s11235-004-5017-x

5. Business Advisory Support: The Growing Popularity of Hosted VoIP (2013). Available at http://www.advisorysupport.co.uk/ the-growing-popularity-of-hosted-voip/ Accessed on 5 July 2013.

6. Cano, M.D., Cerdan, F.: Subjective qoe analysis of voip applications in a wireless campus environment. Telecom- 
munication Systems 49(1), 5-15 (2012). DOI 10.1007/ s11235-010-9348-5. URL http://dx.doi.org/10.1007/ s11235-010-9348-5

7. Cardeal, S., Neves, F., Soares, S., Tavares, F., Assuncao, P.: Arqos (B): System to monitor qos/qoe in voip. In: EUROCON - International Conference on Computer as a Tool (EUROCON), 2011 IEEE, pp. 1-2 (2011). DOI 10.1109/EUROCON.2011.5929310

8. Ciszkowski, T., Mazurczyk, W., Kotulski, Z., Hofeld, T., Fiedler, M., Collange, D.: Towards quality of experiencebased reputation models for future web service provisioning. Telecommunication Systems 51(4), 283-295 (2012). DOI 10.1007/s11235-011-9435-2. URL http://dx.doi. org/10.1007/s11235-011-9435-2

9. De Pessemier, T., De Moor, K., Joseph, W., De Marez, L., Martens, L.: Quantifying subjective quality evaluations for mobile video watching in a semi-living lab context. Broadcasting, IEEE Transactions on 58(4), 580-589 (2012). DOI 10.1109/TBC.2012.2199590

10. De Pessemier, T., De Moor, K., Joseph, W., De Marez, L., Martens, L.: Quantifying the influence of rebuffering interruptions on the user's quality of experience during mobile video watching. Broadcasting, IEEE Transactions on 59(1), 47-61 (2013). DOI 10.1109/TBC.2012.2220231

11. De Pessemier, T., De Moor, K., Ketykó, I., Joseph, W., De Marez, L., Martens, L.: Investigating the influence of qos on personal evaluation behaviour in a mobile context. Multimedia Tools and Applications 57(2), 335-358 (2012). DOI 10.1007/s11042-010-0712-y. URL http://dx.doi.org/10.1007/s11042-010-0712-y

12. Geerts, D., De Moor, K., Ketykó, I., Jacobs, A., Van den Bergh, J., Joseph, W., Martens, L., De Marez, L.: Linking an integrated framework with appropriate methods for measuring qoe. In: Quality of Multimedia Experience (QoMEX), 2010 Second International Workshop on, pp. 158-163 (2010). DOI 10.1109/QOMEX.2010.5516292

13. Giannakidou, E., Koutsonikola, V., Vakali, A., Kompatsiaris, I.: In \& out zooming on time-aware user/tag clusters. Journal of Intelligent Information Systems 38(3), 685-708 (2012). DOI 10.1007/s10844-011-0173-4. URL http://dx.doi.org/10.1007/s10844-011-0173-4

14. Hoßfeld, T., Binzenhfer, A.: Analysis of skype voip traffic in umts: End-to-end qos and qoe measurements. Computer Networks 52(3), 650 - 666 (2008). DOI http://dx.doi.org/10.1016/j.comnet.2007. 10.008. URL http://www.sciencedirect.com/science/ article/pii/S138912860700299X

15. Huang, T.Y., Huang, P., Chen, K.T., Wang, P.J.: Could skype be more satisfying? a qoe-centric study of the fec mechanism in an internet-scale voip system. Network, IEEE 24(2), 42-48 (2010). DOI 10.1109/MNET.2010. 5430143

16. International Telecommunication Union: Definition of Quality of Experience (QoE). Liaison statement, ITU$\mathrm{T}$, International Telecommunication Union (2007). Ref.: TD 109 rev 2 (PLEN/12)

17. Jammeh, E., Mkwawa, I., Khan, A., Goudarzi, M., Sun, L., Ifeachor, E.: Quality of experience (qoe) driven adaptation scheme for voice/video over ip. Telecommunication Systems 49(1), 99-111 (2012). DOI 10.1007/ s11235-010-9356-5. URL http://dx.doi.org/10.1007/ s11235-010-9356-5

18. Jelassi, S., Youssef, H., Hoene, C., Pujolle, G.: Singleended parametric voicing-aware models for live assessment of packetized voip conversations. Telecommunication Systems 49(1), 17-34 (2012). DOI 10.1007/ s11235-010-9350-y. URL http://dx.doi.org/10.1007/ s11235-010-9350-y

19. Karapanos, E., Zimmerman, J., Forlizzi, J., Martens, J.B.: User experience over time: an initial framework. In: Proceedings of the SIGCHI Conference on Human Factors in Computing Systems, CHI '09, pp. 729-738. ACM, New York, NY, USA (2009). DOI 10.1145/1518701. 1518814. URL http://doi.acm.org/10.1145/1518701. 1518814

20. Kutner, M.H., Nachtsheim, C.J., Neter, J., Li, W.: Applied Linear Statistical Models, fifth edn. McGraw-Hill (2005)

21. Lambrinos, L., Djouvas, C.: Improving quality of experience in wireless voip through novel call scheduling. Telecommunication Systems 52(4), 1905-1916 (2013). DOI 10.1007/s11235-011-9473-9. URL http://dx.doi. org/10.1007/s11235-011-9473-9

22. Li, X., Toseef, U., Dulas, D., Bigos, W., Grg, C., TimmGiel, A., Klug, A.: Dimensioning of the lte access network. Telecommunication Systems 52(4), 2637-2654 (2013). DOI 10.1007/s11235-011-9593-2. URL http: //dx.doi.org/10.1007/s11235-011-9593-2

23. Llamas, R., Reith, R., Shirer, M.: Apple Cedes Market Share in Smartphone Operating System Market as Android Surges and Windows Phone Gains, According to IDC. IDC - Press Release (2013). Available at http://www.idc.com/getdoc.jsp? containerId= prUS24257413 Accessed on 26 August 2013.

24. Manousos, M., Apostolacos, S., Grammatikakis, I., Mexis, D., Kagklis, D., Sykas, E.: Voice-quality monitoring and control for voip. Internet Computing, IEEE 9(4), 35-42 (2005). DOI 10.1109/MIC.2005.92

25. Novkov, L., tepnkov, O.: Visualization of trends using radviz. Journal of Intelligent Information Systems 37(3), 355-369 (2011). DOI 10.1007/s10844-011-0157-4. URL http://dx.doi.org/10.1007/s10844-011-0157-4

26. Palmieri, F.: Large scale voice over ip experiences on high performance intranets. In: S. Chaudhuri, S. Das, H. Paul, S. Tirthapura (eds.) Distributed Computing and Networking, Lecture Notes in Computer Science, vol. 4308, pp. 355-366. Springer Berlin Heidelberg (2006)

27. Princeton University: Pareto distribution (2013). Available at http://www.princeton.edu/ achaney/tmve/ wiki100k/docs/Pareto_distribution.html Accessed on 27 August 2013

28. Rajaraman, S.: Five Stars Dominate Ratings. YouTube Official Blog (2009). Available at http://youtube-global.blogspot.be/2009/09/ five-stars-dominate-ratings.html Accessed on 15 July 2013.

29. Reiter, U.: Overall perceived audiovisual quality - what people pay attention to. In: IEEE 15th International Symposium on Consumer Electronics 2011 (ISCE), pp. 513-517 (2011). DOI 10.1109/ISCE.2011.5973883

30. Rowe, L.A., Jain, R.: Acm sigmm retreat report on future directions in multimedia research. ACM Transactions on Multimedia Computing, Communications, and Applications 1(1), 3-13 (2005). DOI 10.1145/1047936.1047938. URL http://doi.acm.org/10.1145/1047936.1047938

31. Soldani, D., Li, M., Cuny, R.: QoS and QoE Management in UMTS Cellular Systems, pp. i-xxvii. John Wiley \& Sons, Ltd (2006) 